\title{
Septic Arthritis of the Hip in an Immune Competent Adult: The Significance of the Differential Diagnosis
}

\author{
Matthew O. Barrett, MD, and B. Sonny Bal, MD
}

We present an unusual case in which hip sepsis occurred in a healthy adult. This case emphasizes the necessity of a meticulous history and physical examination and the mandatory formulation of a differential diagnosis.

\section{Case Report}

A 37-year-old Caucasian female in otherwise unremarkable health presented with left hip and back pain. Symptoms began after a fall 12 months earlier, with worsening hip pain over the past 2 months. At the time of her fall, the patient did not consult a physician and reported superficial bruises and aches that improved without medical intervention.

The patient described pain in her left groin area, but no weight loss, fevers, chills, or sweats. Four weeks earlier, she had seen her primary care physician, and at that time, plain radiographs were interpreted as degenerative joint disease of the hip (Figure 1). She had been referred to our orthopedic service for evaluation for a total hip replacement. The patient's physical examination showed an irritable hip joint with a limited arc of motion. She was afebrile, with unremarkable vital signs. She had an antalgic gait, with clinical findings typical of a degenerative hip joint.

Hip radiographs demonstrated a loss of joint space. The features typically associated with osteo-

\footnotetext{
This article was externally peer reviewed.

Submitted 6 September 2006; revised 5 December 2006; accepted 8 December 2006.

From the Department of Orthopaedic Surgery, University of Missouri School of Medicine, Columbia.

Funding: The authors received no financial support for this study.

Conflict of interest: none declared.

Corresponding author: Matthew O. Barrett, MD, Department of Orthopaedic Surgery, MC 213, DC053.00, 1 Hospital Drive, University of Missouri School of Medicine, Columbia, MO 65201 (E-mail: barrettmat@health.missouri.edu).
}

arthritis, such as subchondral sclerosis, juxtaarticular osteophytes, or cystic degeneration, were absent, and the destructive changes in the hip joint had progressed, compared with the earlier hip radiographs taken by the family physician (Figure 2). Laboratory data showed an erythrocyte sedimentation rate of $59 \mathrm{~mm} / \mathrm{hr}(0-20 \mathrm{~mm} / \mathrm{h})$, and a C-reactive protein level of $1.8 \mathrm{mg} / \mathrm{dL}(0.0-0.9 \mathrm{mg} / \mathrm{dL})$. The serum white blood cell count was 8.6 thousand $/ \mathrm{mm}^{3}$ (4.5-11.0 thousand $\left./ \mathrm{mm}^{3}\right)$. A 3-phase bone scan was considered as the next step, but in light of the patient's young age, atypical clinical presentation, and rapid progression of radiographic changes, an aspiration of the hip joint was performed first. A small amount of blood-tinged fluid was aspirated from the arthritic hip with fluoroscopic guidance, and cultures ultimately confirmed hip sepsis secondary to Staphylococcus aureus. As is occasionally the case with hip aspirates, insufficient fluid was obtained for further synovial fluid analysis. Therefore, cell count and differential were not available to help guide decision making.

\section{Discussion}

This report illustrates the value of considering the differential diagnosis of hip disease when the plain radiographs show rapidly progressive joint space narrowing. Usually, joint space narrowing is associated with osteoarthritis. In the present case, the rapid radiographic progression of joint space destruction and the patient's age led to consideration of alternative diagnoses.

Joint space narrowing in the hip can be secondary to osteoarthritis, sepsis, inflammatory arthritides, pigmented villonodular synovitis, bleeding disorders, synovial hemangioma, and chronic trauma. ${ }^{1-3}$ When symptoms develop or worsen acutely in association with rapid radiographic deterioration of the hip joint, the diagnostic possibili- 


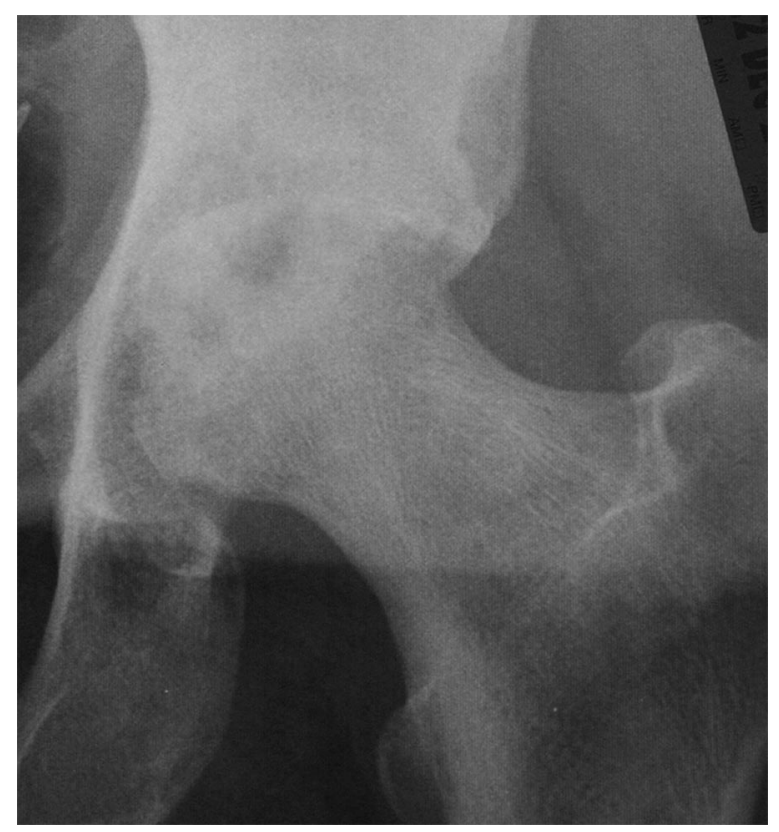

Figure 1. Anteriorposterior radiograph of the hip taken 4 weeks before the image shown in Figure 2, with changes that were interpreted as degenerative joint disease.

ties can include septic arthritis, rheumatoid arthritis, osteonecrosis of the femoral head, neuropathic arthropathy of the hip, malignancy, seronegative arthropathy, and rapidly destructive osteoarthropathy of the hip joint. ${ }^{4,5}$ The radiographic presentation of hip sepsis can be very similar to that of rapidly destructive osteoarthropathy of the hip. Aspiration of the hip can distinguish between the 2 entities, and is recommended if the clinician encounters rapid radiographic destruction of the hip joint. ${ }^{6}$ Although culture of the aspirate is crucial, further synovial fluid analyses-especially cell count and differential— can facilitate the diagnosis. Septic arthritis generally exhibits white blood cell counts in the range of $80,000 / \mathrm{mm}^{3}$ or greater, whereas a noninflammatory arthritic effusion is usually less than $2000 / \mathrm{mm}^{3}$. Glucose and protein are 2 less sensitive and less specific parameters that have been used in the past. ${ }^{7}$ In osteoarthritis, these values are typically equal to that of the serum. Sepsis in the joint generally causes a decrease in joint aspirate glucose and an increase in joint aspirate protein, relative to the serum. ${ }^{8}$

The unusual feature of the present case was that hip sepsis occurred spontaneously in a healthy adult. Septic arthritis of the hip joint in otherwise healthy adults is very unusual. ${ }^{5}$ Septic hips are usu-

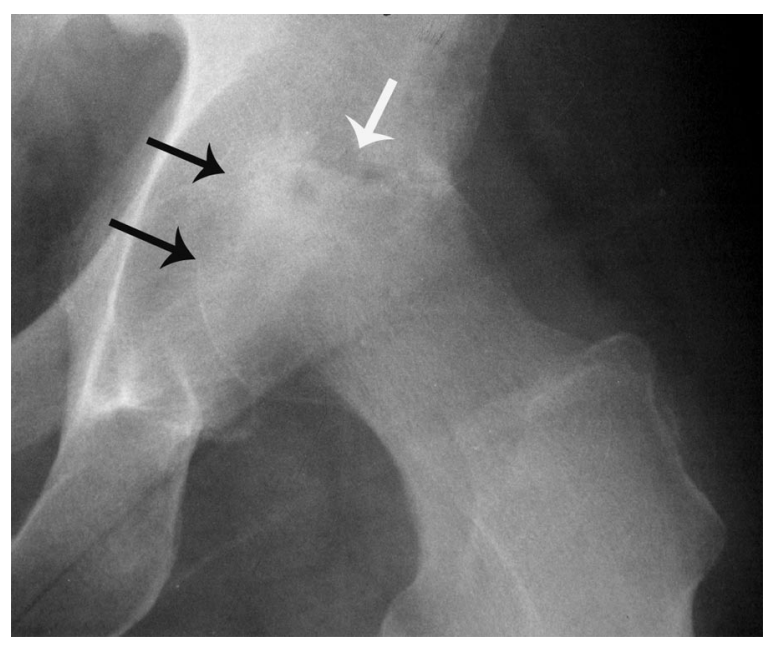

Figure 2. Anteriorposterior radiograph with concentric narrowing of the joint space (black arrows) and progressive lytic changes (white arrow).

ally encountered in the pediatric population or in adults with deficient immune systems. Risk factors such as diabetes mellitus, rheumatoid arthritis, steroid therapy, alcoholism, chronic liver or kidney disease, cancer, malnutrition, and immune deficiencies are associated with septic arthritis of the hip. Patients with sickle cell anemia also have a documented susceptibility to joint sepsis. ${ }^{9}$ Rarely, spontaneous nongonococcal and Candida sepsis ${ }^{10}$ of the hip joint in otherwise healthy adults have been reported. Posttraumatic bleeding into the hip joint followed by bacterial seeding of the hematoma may explain why sepsis occurred in the present case, because the patient had fallen several months previously. ${ }^{5}$

Staphylococcus aureus continues to be the most common organism isolated from adult hips affected by sepsis. ${ }^{5}$ Gonococcal septic hip arthritis is less common, and it is usually associated with specific host risk factors. ${ }^{11,12}$ Direct extension from an intraabdominal focus via the psoas and inoculation after femoral venipuncture ${ }^{13}$ are other unusual and rare etiologies of hip sepsis.

\section{Conclusion}

If the patient had undergone a total hip replacement in a septic environment, the outcome would have been disastrous. The primary care physician as well as the surgeon must remain vigilant even with what may seem mundane. Stepwise patient evaluation and critical review of the hip radiographs- 
followed by consideration of the radiologic and clinical differential diagnoses-can lead to the correct diagnosis and treatment in patients with progressive hip degeneration on plain radiographs. The radiologist seldom has as much clinical information and perspective as the treating physician. Therefore, radiographs should optimally be reviewed in person by the clinician if possible.

Once the diagnosis of sepsis had been confirmed in this patient via aspiration, she underwent irrigation and debridement of the hip joint. A temporary implant loaded with antibiotics was placed to maintain the hip joint and clear the infection. Ultimately, after the infection cleared, she underwent uncomplicated total hip arthroplasty and has subsequently had an excellent clinical result at 2-year follow-up.

\section{References}

1. Abrahams TG, Pavlov H, Bansal M, Bullough P. Concentric joint space narrowing of the hip associated with hemosiderotic synovitis (HS) including pigmented villonodular synovitis (PVNS). Skeletal Radiol 1988;17:37-45.

2. Cotten A, Flipo RM, Chastanet P, et al. Pigmented villonodular synovitis of the hip: review of radiographic features in 58 patients. Skeletal Radiol 1995; 24:1-6.

3. Dwosh IL, Resnick D, Becker MA. Hip involvement in ankylosing spondylitis. Arthritis Rheum 1976;19: 683-92.

4. Bock GW, Garcia A, Weisman MH, et al. Rapidly destructive hip disease: clinical and imaging abnormalities. Radiology 1993;186:461-6.

5. Bulmer JH. Septic arthritis of the hip in adults. J Bone Joint Surg Br 1966;48:289-98.

6. Khanna AJ, Domb BG, Moshirfar A, Wenz JF Sr. Rapidly destructive osteoarthropathy of the hip. Am J Orthop 2004;33:243-47.

7. Shmerling RH, Delbanco TL, Tosteson AN, Trentham DE. Synovial fluid tests. What should be ordered? JAMA 1990;264:1009-14.

8. Brinker MR, Miller MD. Fundamentals of Orthopaedics. Philadelphia: W.B. Saunders; 1999.

9. Bennett OM, Namnyak SS. Bone and joint manifestations of sickle cell anaemia. J Bone Joint Surg Br 1990;72:494-9.

10. Kawanabe K, Hayashi H, Miyamoto M, et al. Candida septic arthritis of the hip in a young patient without predisposing factors. J Bone Joint Surg Br 2003;85:734-5.

11. Lee AH, Chin AE, Ramanujam T, et al. Gonococcal septic arthritis of the hip. J Rheumatol 1991;18: 1932-3.

12. Tindall EA, Regan-Smith MG. Gonococcal osteomyelitis complicating septic arthritis. JAMA 1983; 250:2671-3.

13. Fromm SE, Toohey JS. Septic arthritis of the hip in an adult following repeated femoral venipuncture. Orthopedics 1996;19:1047-8. 